\title{
Neuer Therapiestandard in Sicht
}

\author{
Erstmals seit Jahren konnte \\ wieder ein relevanter Fortschritt \\ in puncto Ansprech- und Überle- \\ bensrate bei der Behandlung des \\ metastasierten Pankreaskarzi- \\ noms (mPCa) erzielt werden.
}

60-70\% der Patienten mit PCa haben zum Zeitpunkt der Diagnosestellung bereits Metastasen, berichtete Volker Heinemann, München. Die Kombination von nab-Paclitaxel (Abraxane ${ }^{\circledast}$ ) plus Gemcitabin (GEM) in der Erstlinientherapie des mPCa wurde jetzt in der internationalen Studie MPACT (Metastatic Pancreatic Adenocarcinoma Clinical Trial) mit dem aktuellen Standard GEM in Kombination mit nab-Paclitaxel verglichen. Insgesamt 861 neu diagnostizierte, nicht vorbehandelte MPA-Patienten erhielten randomisiert entweder $1.000 \mathrm{mg} / \mathrm{m}^{2} \mathrm{GEM}$ plus 125 $\mathrm{mg} / \mathrm{m}^{2}$ nab-Paclitaxel oder eine GEMMonotherapie.

Das Ergebnis zeigte in allen Subgruppen eine signifikante Überlegenheit der nab-Paclitaxel-Kombination hinsichtlich des primären Endpunkts Gesamtüberleben (8,5 vs. 6,7 Monate). Darüber hinaus wurde ein Anstieg des 1-Jahres-Überlebens ( 35 vs. $22 \%$ ) sowie eine deutliche Verbesserung der Ansprechraten (23 vs. $7 \%$ ) und des progressionsfreien Intervalls $(5,5$ vs. 3,7 Monate) erreicht. Als positiv bewertete Heinemann das akzeptable Nebenwirkungsprofil des Regimes. So lag die Neutropenierate mit $38 \%$ deutlich niedriger als erwartet; die periphere Neuropathie (17\%) bildete sich am Ende der Therapie relativ schnell zurück.

Der Experte sieht in nab-Paclitaxel plus Gemcitabin künftig einen neuen Therapiestandard bei MPA. Aufgrund der positiven Studienergebnisse erwartet er die baldige Zulassung von nab-Paclitaxel in dieser Indikation. Das Taxan ist derzeit bereits für die Behandlung des metastasierten Mammakarzinoms zugelassen.

Martina-Jasmin Utzt

Fachpressegespräch "nab-Paclitaxel beim fortgeschrittenen Pankreaskarzinom - aktuelle Daten vom ASCO-GI", München, 31.01.2013; Veranstalter: Celgene

\section{Dauerhaft tiefes Ansprechen erreichen}

\author{
Auf der 54. Jahrestagung der American Society of Hematology (ASH) im \\ Dezember 2012 vorgestellte Studiendaten bestätigen eine hohe Wirksam- \\ keit von Nilotinib bei der chronischen myeloischen Leukämie (CML).
}

\begin{abstract}
4-Jahres-Daten der Phase-III-Studie ENESTnd (Evaluating Nilotinib Efficacy and Safety in Clinical Trials of Newly Diagnosed $\mathrm{Ph}+\mathrm{CML}$ Patients) zeigen, dass bei neudiagnostizierten Patienten mit CML in der chronischen Phase Nilotinib (Tasigna ${ }^{\circledast}$ ) Imatinib deutlich überlegen ist. Unter Nilotinib $(600 \mathrm{mg} / \mathrm{d})$ erreichten si-
\end{abstract}

gleich zu Imatinib während der Studie signifikant geringer (0,7 bzw. 1,1 vs. $4,2 \%)$ und kein Patient, der eine MR4,5 erreichte, erlitt einen Progress. Darüber hinaus zeigte die auf dem ASH ebenfalls vorgestellte ENESTnd Landmark-Analyse, dass ein schnelles und tiefes molekulares Ansprechen nach drei Monaten prädiktiv für das Erreichen tiefer molekularer Remissionen, der Progressionsfreiheit sowie einem Überlebensvorteil ist [Hochhaus A et al. ASH 2012; Abstr. 167]. Das akzeptable Verträglichkeitsprofil von Nilotinib wurde in den 4-Jahres-Daten dieser Studie erneut bestätigt.

red

Nach Informationen von Novartis

\section{Individuelle statt schematische Nachsorge}

\section{Nachsorge nach Schema F ist nicht immer von Vorteil.}

Für die Nachsorge wurden bislang als Ziele u.a. standardisierte Nachsorgeschemata, Früherkennung von Fernmetastasen und Krankheitsfrüherkennung definiert, berichtete Ulrich R. Kleeberg, Hamburg. Die Früherkennung von Fernmetastasen habe aber keinen Einfluss auf die Heilungsrate: Trotzdem betrage z. B. die Le- benserwartung der Patientinnen mit metastasiertem Mammakarzinom unverändert nur zwei Jahre. Durch die Früherkennung, z.B. bei Bestätigung von asymptomatischen Metastasen, könne sich die Lebensqualität der Patienten sogar verschlechtern. Beobachtung sei deshalb bei vielen Patienten sinnvoller als eine schematisierte Nachsorge. Notwendig sei daher die individuelle Gestaltung der Nachsorge: eine retrospektive Aufarbei- tung der Krebserkrankung und eine prospektive Lebensplanung. Umsetzbar seien die Ziele Bewegung und iso-/hypokalorische Ernährung. Wegen des begrenzten Nutzens für Patienten mit metastasierten Tumorerkrankungen empfiehlt Kleeberg Zurückhaltung bei diagnostischen und therapeutischen Interventionen. Entscheidend für die somatische und psychische Situation der Tumorpatienten sei ihre Lebensführung.

Friederike Holthausen

Satellitensymposium „Agenda 2020: Aktuelle Herausforderungen im Kampf gegen Krebs", DGHO-Jahrestagung 2012, Stuttgart, 19.10.2012; Veranstalter: Bristol-Myers Squibb 